\title{
Data Culture Generation: After Content, Process as Aesthetic
}

\author{
Alistair Riddell
}

\section{THE BOULEVARD IN ASPECT}

This essay assumes that the reader has at least an understanding of contemporary meanings of "process" and "data." Any reasonable contact with recent technology should have set up fundamental, if intuitive, definitions and relations even if experienced through a highly pragmatic context. While the underlying praxis of computer programs, applications and systems are critical for an understanding of this essay, "process," "data" and "information" are, of course, effectively timeless, profound and multi-faceted human concerns. It is simply their manifestations that have changed. Aware of this from the outset, the reader should not be surprised that the terms will be thought through as broadly as possible, including those less-easily codified creative dimensions that surround the methods and means to generate artistic results or products.

The notion of "process" is intriguing because it is increasingly seen as complex and proprietary. Complex because many systems in our societies, crucial to our well-being, are beyond the understanding of any one person at any one time. Additionally, there are intangible aspects or side-effects of "process" and "data." In this age, when process implies production, the value of the "process," often independent of the "data," has increased, as it is almost universally understood as an efficient means to generate or "process" more than one unique item (or datum). Mass production is thus an essential activity for our society, and the concept has not been lost on the art world. The democratization of art has necessitated that, where possible, creative works be duplicated or, more usually, where this is not possible, the memory of their power (aura) be made available to as many people as might desire it. Here we see the rise of information transfer or the conveyance of the power of the artwork as a critical activity in the cultural world. And all of this can be highly influenced by the machinations of marketing and the vagaries of consumer demand.

Data is initially considered that which a (computational) process or application works on or with, inputs or outputs; it has, at times, various values. If the audio signal from a compact disc (CD) appears to have no immediately discernible flaws, it is regarded as valuable and correct. The implication is that this is what the producer of the CD intended for public presentation. If a CD appears flawed, then that must be explained either as an aberration, if other CDs do not contain the same condition, or part of the producer's intention. This is usually verified at the point of sale, but uniqueness and rarity may actually increase the value of the CD under certain circumstances. To convert a flaw into something of artistic value requires authority or a condition that itself must be ratified by some special process. Such processes can occur on a very large cultural scale, even when the data is not flawed.

One such special process is sampling. A sample or distinctive fragment of another artist's music, used in a new and other artist's work, while not a flaw or unintentional error, constitutes a radical rethinking of musical process. The use of samples is now a widely accepted and occasionally illegal practice that has as its basis of authority an arguable position of artistic license founded on the premise of a new aesthetic. Not only is the content exploited but also the performance and recording ambience, which can add to a new work an artificial historicism. The effect can be blatantly obvious or profoundly subtle but is rarely without controversy, which itself is a much sought-after aesthetic quality. Consider that much to James Brown's horror-and the detriment of his bank account-he turned out to be one of the most unremunerated of the sampled artists of the 1990s, whose sampled music, ironically, contributed to the legitimization of sampling as powerful cultural practice. Surrounding the flagrant breaches of copyright were artistic statements granted a tacit but nevertheless effective authority through a youth culture "hip" to breaches of the law in the search for its own Zeitgeist statement.

This preamble takes a simple view of the terms it discusses, but it should demonstrate that the topic itself is worthy of extended inquiry. Examples of "data" and "process" abound but they invariably suggest exceptions and variations that challenge any general understanding and will probably continue to do so as we move into more abstract investigations.

Some explanation of the present state of contemporary culture would seem an appropriate beginning to an investigation of the influence of process and data on contemporary musical culture. A discussion that freezes the moment and explains the constituents and their place in the scheme of things would seem perfectly reasonable, if it were not for the fact that of interest here are the running engines of change and the nature of the data flow. Leonard Meyer, with music in mind, sets the scene:

Alistair Riddell (artist), 12 Napier Street, Fitzroy, Victoria 3065, Australia. E-mail: $<$ amr@alphalink.com.au>.

Based on a paper presented at First Iteration, a conference on generative systems in the electronic arts, 1-3 December 1999, at Monash University in Melbourne, Australia. 
During the past seventy-five years or so, the ideology of Western-world culture has been undergoing a profound change. Barely discernible at first, this change has increasingly affected choice in many realms of our culture The change is not about the principles governing the natural world, about the essence of human nature, or about the bases of human cultures (though it interacts with all of these). Rather the change is about the nature of change itself. More specifically, the change has to do with our culture's beliefs about the future and therefore about its understanding of the present as well [1].

The above paragraph introduces an analysis of contemporary musical culture that was begun a quarter of a century earlier. Much of what Meyer's succinct closure illuminates is already implicitly understood or felt by most of us now, privately acknowledged in our expectations and engagements. We haven't arrived at this behavior unaided and without some kind of empowerment. We have used a new kind of technology. We have placed this technology in a position that guarantees not only change but a momentum for change as well. Meyer describes this as a "fluctuating stasis." Living in this state means that the forces changing music no longer have the appearance of a singular direction that once characterized Western music as little as 30 years ago. More significantly, rather than pondering this concept, we are now more interested in the experience of living and negotiating our lives through it.

Meyer's explanation, initially intriguing in its broad sweep, eventually begs for some kind of confirmation in local or current detail. We need to conduct "spot checks" to see if the condition still holds true. A simple examination of local life and art should suffice-a sort of sniffing at the air, so to speak. How we conduct this examination depends upon our ability to correlate activities in our backyard with what we understand on the broader cultural scenario. In the past 30 years, many musical instances have been documented in ways that have brought to the attention of almost all musicians and the wider public the idea that musical thinking and practice is informed by the vast social dimension of contemporary life, with the aid of various technologies, and in ways and for reasons that have been difficult to document from a traditional pedagogical position.

Musical interest in process has intensified this century with the progressive "electrification" of sound. From transfer, recording and synthesis to the current aspirations of the digital signal environment, technology bestows pragmatic credence and relocates musical activity closer to the abstraction of science and mathematics. The computer, in this respect, is the instrument par excellence, inspiring and instigating the idea of a scientific/technological art unbounded in the present and future while simultaneously encapsulating and concentrating activity in a focused, highly structured and codified context.

The idea of accumulated knowledge and theory has changed. That the future of music can draw upon a theory at all is less likely than it was half a century ago. Yet any music system that includes a code of usage with, possibly, a hidden aesthetic, constitutes a music theory. Continued usage of the system finds the music and its practice and results ingrained with behavior and patterning. What we have today is a proliferation of music software that acts as "theories" and quite different in cognitive influence from traditional musical theories. If the user does not tacitly agree to abide by the formal usage and, to a lesser extent, the artistic focus of the system, then the anticipated results become less predictable. This, I think, is implicit in computer music systems. Certainly, the systems will not spontaneously tell you how to write good music nor are they particularly interested in imposing style. What they offer is a kind of conceptual framework and a sense of community. One doesn't need to know all or any of the other users, but if you wish to, they are probably out there, using the system in much the same way.

Consider Milton Babbitt's work with the synthesizer in the late 1950 s and 1960 s, work now enshrined in the annals of history [2]. He used the RCA system to implement serial ideas. Computers were then far from being anything close to a musical instrument.

The knowledge required to run the system was intensely arcane; it was a protected world with limited access. Babbitt's musical results, though highly interesting, reflected this lack of access and only addressed themselves to a community of specialists. It would be another decade before the role of the computer in musical creation could be viewed as useful and desirable.

Compare Babbitt's activities with those of Lejaren Hiller and Leonard Isaacson, who, at about the same time, were pursuing computer-composed instrumental music. This resulted in the now famous
Illiac Suite for String Quartet [3]. One could see this as an effort to reassert the familiar by means of the new. Electronic music has been challenged by this dichotomy in artistic practice ever since. Using computers to write music for string quartet and pursuing traditional instrumental aspirations in electronic sound immediately raised questions about the displacement or replacement of human activity in the manifestation of music. Since that time the question of what technology should be used for, with particular reference to a future music, has become more or less irrelevant. Music technology has simply evolved to accommodate almost any musical aspiration that an artist might have, simultaneously supporting traditional and non-traditional ways of thinking about music.

The 1960s world of Marshall McLuhan and John Cage, as eloquently assembled by James Pritchett in his book on Cage, brings into focus Cage's considerations of music in the information age. With hindsight, Cage's understanding of and musical response to McLuhan's ideas seems remarkably more farsighted than many of the electronic works produced by some of his contemporaries, from a purely musical perspective.

\section{Pritchett writes:}

McLuhan did more than provide an explanation of the changes in society: he affirmed the primacy of the artist in dealing with those changes. Because the artist deals with the senses, he is best equipped to understand the changes in perception caused by new technology. McLuhan saw experimental artists such as Cage as prophets of the new technology, and offered them a new purpose: the instruction of society on "how to rearrange one's psyche in order to anticipate the next blow from our own extended faculties." This is the point at which Cage and McLuhan converge: Cage had always seen music as a means of "changing one's mind," and McLuhan provided a good reason to change it [4]

And again, quoting McLuhan: "Any technology gradually creates a totally new human environment. Environments are not passive wrappings but active processes" [5].

Cage responded:

We are now, McLuhan tells us, no longer separate from this environment. New art and music do not communicate an individual's conceptions of ordered structures, but they implement processes which are, as are our daily lives, opportunities for perception (observation and listening). McLuhan emphasizes this shift from life done for us to life that we do for ourselves [6]. 
Not all contributions to an understanding of the musical future in the second half of this century have had such a public or creative exposure as those of John Cage. The musical and intellectual efforts of Pierre Schaeffer remain shrouded in his musical genre, musique concrète, and his philosophical writings. His attempt at a treatise on sound (or the sonic object) was a bold and incomplete project. Even more ambitious was his research into a typology and morphology of sounds. This undertaking was never effectively completed, largely because it raised too many irreconcilable issues. Nevertheless, the concept had an enormous influence on many composers, both in the electronic and traditional musical worlds. His musical works are perhaps more compelling today because of the relation to digital audio possibilities now widely available to musicians. Carlos Palombini's text on Schaeffer's work not only provides a contemporary insight into the music but a complex analysis of Schaeffer's aspirations and effect.

Of Schaeffer's legacy, Palombini writes in his conclusion:

Beyond semiotics, there is another realm where Schaeffer has done spadework, not the least for his attempt to distance himself vis-à-vis particular aesthetics. I am referring to the creation of digital systems for musical synthesis and composition. The whole research carried out by Schaeffer into the role the notion of instrument has played in music . . . is invaluable. I have pointed out the paradox whereby classificatory typology selects sounds which are suitable to musicality when a musical langue does not exist or has been abolished by deconditioning (rather than by division of classes). Is this not the paradox every digital luthier comes face to face with? [7]

Cage and Schaeffer, in their texts and music, display not only different perspectives and uses of technology but different philosophies. Cage was challenged by and responded to technology as something quite esoteric, abstract and spiritual, whereas Schaeffer engaged technology with a pragmatic, humanist mind. It is interesting to note that technological interaction ultimately inspires a desire for formal definitions. Although Cage worked with various technologies (tape recording, radios and computers), he seems to have avoided becoming focused on any one technology to the exclusion of a broadly based creative curiosity. Schaeffer, on the contrary, through his focus on recorded sound, seems to have become fascinated by issues emerging from his prolonged contact with the technology.
There are composers for whom technology inspires a singularly brief flourish of activity, during which they focus on a creative issue that they hope technology, or at least technological thinking, might illuminate. In the early 1990s, Eliot Handelman described in his Ph.D. thesis an experience in Berlin in the mid-1980s, when the Swiss composer/instrument maker Walter Smetak gave a concert that was to include a preamble describing his "instrument of silence" (silencarium) [8]. Handelman, who at the time was studying orchestral composition, remarked that the concert began with a discussion of the composer's repertoire of instruments and a slide show, but proceeded so slowly that the hour grew late, and consequently the description of the silencarium was passed over. No description of "the instrument of silence" was given. Handelman concluded that as far as one could make out from the slide, the instrument "seemed to be quite elaborate, a far from obvious realization of a unique idea." While this could have been an update on Cage's 4'33, the feeling was that something more substantial was needed to produce silence. Pure silence is, of course, extremely difficult to produce, because it requires the antithesis of action, and what process would negate action?

Handelman's thesis, Music as Secondary Consciousness: An Implementation, postulated an even more radical leap in the nature of musical evolution. While it contained discussions on music and consciousness, concluding with a series of LISP programs that modeled listening modalities as he conceived them, it explored the questions of what it is to be a "listener" and what it is for music to be the instrument for the transmission of mind. Handelman wrote:

This essay concerns my vision of the future of music, or to be more precise, a future that will have had a musical past. I conceive of music as a medium whose proper concern is the transmission of mind, or, as I prefer to call it, the simulation of consciousness. I want music to develop in such a way as to become the technological instrument of this transmission [9].

The thesis, in and of itself, is a composition statement. The thesis contributes to the emergence of a practice of producing musical "thought experiments" encapsulated in complex contexts and processes through the use of contemporary technology. Handelman's thesis is a commencement for a line of inquiry that suggests a relationship between music, technology and the mind that has not been explored before. There are no real tangible theories or substantial results here, rather a trail of historical and contemporary ideas that together form an interesting proposal about the future of music.

\section{MODES OF PRODUCTION}

Away from the historical, the well-documented and the musical world of individuals, tapering as it does to this moment, we find ourselves in a less-documented and yet well-encoded space. Technology's impact on music can now be likened to an exploded supernova. During the long blinding moment, it is hard to see the detail of an increasingly diverse and extreme musical environment. The 1980s marked the period when digital technology seriously entered the musical milieu, signaling innovation and a musical future for everyone. Curiously, that musical moment also seemed to implode, with the resurgence of vinyl records as the medium of the DJ and the beginning of an epoch of complex musical aspirations. Appearing rather anachronistic at first, this form of recorded music presentation created a new type of instrumentalist and turned upside-down the idea that recording is simply an archival medium. Turntables became instruments, DJs virtuosi. Threeto five-hour sets pushed music as a stream-of-consciousness minimalism to new frontiers, and tens of thousands of young people rallied to the art. New technologies quickly began playing an important role in sustaining production for this musical market.

The skills of DJs have increased dramatically in the last 10 years. Performances are as complex as in any other musical practice that has arisen exclusively from a youth-culture environment. Various techniques have to be learnt and a particular aesthetic must be mastered in order for a DJ to distinguish him- or herself from thousands of other DJs.

DJs and dance cultures have had another impact on music. They have created a specialist market for new music that needed to be produced on a regular basis, with a degree of innovation and originality that would ensure the evolution and survival of the musical milieu. Kodwo Eshun gives a detailed account of the dynamic evolution of the techno fringe dwellers Basic Channel/Chain Reaction in Berlin in the late 1990s [10]. This kind of musical community/industry is complex but by no means exceptional of the vast musical underground, where music is produced in relatively 
small quantities, by hundreds of specialist producers with connections to important musical contexts. Although the music may be accessible at a conventional retail level, it is also aimed at professionals and those with more than a passing interest. Chain Reaction is also representative of spin-off music genres that are intended as a listening experience rather than dancing activity, but the boundary is often blurred.

While vinyl records, DJs and dance culture challenged notions of conventional musical production, another group of artists were coming to the realization that process, once partially automated or made self-contained, might constitute a performance viewable at any time by a sporadic audience. Although the concept of the installation dates back to the great exhibitions of the last century, this century has made the installation an art form. As a complex spectacle, it presents an idea or concept that is to be experienced through place, image and sound. It is an ideal medium for the presentation of complex, technologically based concepts that require a degree of acquiescence on the part of the receiver. Depending on the context, it can be extremely personal or communal on a physical and more intimate level than most musical or theatrical performances.

The rise of the art of the installation has been driven by a new pragmatism in the arts that seeks to address certain financial, cultural and performance concerns that the presentation of either music or sculpture alone does not fulfill. Installations can be fully operational when the artist is not around and respond to different conditions throughout long presentation periods. They can convey a creative message as effectively as traditional art, perhaps without making explicit and traditional demands on an audience. Installations are for a generation of audiences accustomed to a certain form of spectacle and who want their sensory/emotional/mind-stirring didactic experiences to be brief, exciting and discretionary.

Installations externalize the idea of process and frequently mirror a society's attitude to content and experience. Installations are widely used in retail advertising, product promotion and various cultural events (sport, trade and agricultural shows). Everyone has experienced an installation in one form or another. The difference in the art context is the nature of the message. Without a reflective context, installations tend towards base entertainment with a message that restates the obvious. Nevertheless, installations are changing the way we think about sound and what we expect music to be.

Although computer technology has pursued the dream of real-time operation and largely achieved it, there are several factors that suggest that this ambition is now rather dated. The proliferation of technology over the last 15 years that provided the momentum for electronic and computer music production has brought the means of musical production to thousands of musicians. Together with demands for recordings of DJs, music as organized sound is available in such vast quantities that it no longer can be easily assessed or even adequately listened to. To evaluate music at a physical or tangible level, that is, how we relate to the sound, how the sound was produced or what various sounds signify, is increasingly difficult as the ability to trace and understand the primary process of composition retreats into technical obscurity. A work on CD may have an impressive surface and speak powerfully and economically to the Zeitgeist, but unless you know what technology is being used, the connection between the music and the composer's actions can be non-existent. All there is, is the sound. Some argue that this is the way music should be; others disagree. It would appear to depend on whether the idea of a private music is preferred over a communal one.

The widespread acceptance of music technology has largely been based on the fact that it addresses the most basic and conservative of musical elements and praxes. MIDI, for example, perpetuates the idea of the "note" in an age that has just managed to liberate sound from notation-while remembering that the words "note" and "notation" are cognate.

Paradoxically, it is the perceived limitations of technology that pervaded much of the early hardware and software that caused artists to say, "Well OK, it only does this. What if we do it in this context or with these sounds or at this end of the range or with external equipment?" In other words, the technical limitations necessitated exploration of the system in non-musical terms. It is the classic scenario of playing with a new piece of equipment and turning all the settings to MAX.

As technology got cheaper and more available, small private studios proliferated. Every room could produce sound. Recordings are now ubiquitous, pouring out everywhere. For those composer/ musicians whose music is taken, distributed and listened to by many people, the music scene would not appear to have changed much. For those who find it hard to get their music out there, alternatives to music must be sought. The traditional position is to develop a "live" act. Invariably this differs from the studio material, forcing a return to traditional music practice and the "live act" context (an ironic proposition if the artists are heavily dependent on recording and computer technology for the presentation of their music live).

A far more radical alternative to the production of sound is the production of musical software. This growth area is further evidence of the desire of musically minded people to make a musical statement that somehow goes beyond sound. Musical ideas when presented as music sometimes meet resistance in the usual forums; but an idea translated into a working piece of code that generates music, or transforms or analyzes it, is often met with much greater interest. Ways of thinking about music that go beyond MIDI, or the note, which offer new musical aesthetics, encoded as software, are surely an exciting point of departure for an open-ended musical future that involves the listener in a participatory gesture not found in the transmission of audio.

Dissemination has become one of the essential problems of music (or software) in recent years. Precipitated by increased production across all styles and ideologies, together with the inability or unwillingness of the music industry to respond but instead bunker down in shock at music too diversely manifold to market, new modes of music distribution have been keenly sought. Almost all are modeled on the paradigm established by the music industry over the past century and consequently dependent on it. However, the Internet has proven a powerful mechanism for challenging notions of music distribution, even if it does not yet offer a really viable solution.

The realistic solution to music distribution on the Internet is not technically in sync with the standard of audio quality in general use. As the prevalent means of transmitting audio on the Internet, MP3 is an inferior form of encoded audio signals constituted in very little data-a very important condition for moving music around the Net. While this must be seen as only an interim measure until either the Internet gets faster or a better compression scheme is found, the effect has nevertheless been 
to create a cult following based on a sense of freedom through the empowerment of distribution. Audio data becomes a fetish, yet is easily disposed of. Availability is preferable to quality. This does in part reflect on the music, but there are also the underlying process of access and the legal issues that make accessing the data enticingly risqué. MP3 is audio seemingly liberated from the tyranny of technology and the controlling hegemony (the music industry). It feels like a "teen spirit" thing. We might anticipate a new music based on reworking MP3 recordings pulled from the Internet, and there already exists a culture of Internet musicians whose music exists only in that form. In this respect, the Internet is more than just a means of distribution, it becomes a raison d'être for a culture based on audio data.

It appears that the ambition of being able to make all music available everywhere is being realized faster than we might have imagined a generation ago. The effect is to pit the production and dissemination of music in the collective consciousness against the privacy and expressive substance of the music itself. Time to understand music below the surface is a luxury that few can afford. This was historically the case for many listeners, and now, given the vast amount of recordings available, the situation does not appear to have changed.

\section{Composing IN PROCESSES}

So far this essay has examined a few aspects of technology that might inspire composing processes that set forth conditions for a music. What does that mean? Since it is difficult or indeed less important to talk about the music, the idea translates as ways of thinking about musical composition as a sequence of events, behaviors, conditions and choices, possibly too complex to understand in a static context. Here too, sound plays a less important role, because it is anticipated that the process will construct interesting aesthetic states from sounds that may in themselves not have suggested such potential. In the area of computer music, this is a challenging proposition. Wide ranges of interpretation and application can be expected, because there are so many variables that can be arbitrarily selected and employed. Use of existing systems, software development or employment of other artists are but a few of the numerous possible permutations. This is the beginning of many choices. How does this relate to music? The indirec- tion, that is, the concern for the schematic rendering of composition systems, is a response to issues of experimentation and exploration in an age of facile musical production. The need to produce a lot of music is tempered by the need to produce interesting music; but, before that, interesting processes offer a kind of intellectual preamble. Maybe the music never appears!

A process need not be a model of any behavior or system other than itself. The intention of modeling actual or perceived external behavior can be completely arbitrary. The completeness and effectiveness of a process may be limited. Just as it is impossible to experience, in any given moment, common musical practices of classical, jazz, pop or dance, so too is it impossible to represent in music all possible permutations of a computational musical process. Much of our understanding of ourselves and our ancestry is constructed from fragments of data. We accept that this could be misleading and, at face value, wrong but it remains important because without it there is nothing upon which we can construct an historical identity.

Many forces are at work shaping thought about and use of contemporary technology. They are often interrelated and evolve out of a base functionality that needs improvement. The Internet is a good example here. The obvious solutions are those that tend towards mass appeal through fashion and personal empowerment. A particular functionality can be developed because it facilitates use at a particular level of engagement and need. More sophisticated needs require more time and thought in order to be realized and are likely to attract little developmental impetus other than from technical curiosity.

Thus the evolutionary rate of technology is variable, depending on the support and demand of the population either as contributors or consumers. In both cases, the momentum is increased when the solution can be initially represented in very simple terms that correspond, in part, to the name of the solution. Donald Norman presents a critical analysis of "information appliances," and it seems clear that the forces operating on the development of these in general must also influence the way we expect to be making music in the technological future. Given the problems with generalpurpose computing, we are definitely influenced by failures in functionality, interface design and confusion in purpose. Norman further states:
Appliances are consumer products, whereas computers are technology products; therein lies the fundamental difference in the market place. Computers emphasize technology, appliances emphasize convenience, ease of use; they downplay or even hide the technology [11].

"Musical appliances" have been around for some time now. In your local music store can be found a miscellany of "electronic" instruments aimed at the novice, which are, however, conceptually dubious in relation to the ambitions of informed artistic production. This doesn't diminish the desire for technology that simplifies certain tasks or the overall production of music. In the case of musical performance or composition, what can be simplified and what is necessarily challenging for a creative end need to be clearly understood.

What is important here is the perception of process as a new frontier (or one constantly re-invented). I can see that the term "appliance" will struggle to find credibility in the creative world because of its base connotations. Apart from a functional level issue, it is uninspiring and tends towards a corporate sensibility (fine, if that is what is required). Still, we cannot really anticipate what might come of the creative use of such "appliances." Through the distribution and dynamic of any processing mechanism (computer or appliance), others may anticipate producing unique sound and musical experiences, typically in ways that were not foreseen. Even if these are very localized, heard by only a few, engagement with the process sets up a shared experience that has less to do with the music and more to do with the aesthetic of activity. This inevitably influences the nature of musical aesthetics. Projects established by Thomas Dolby and Brian Eno have at least seeded the musical environment with notions of musical production based on techniques usually associated with more obscure musical systems.

Having said this, the evolution of a practice based on the development of processes is likely to remain small, marginal and diverse in intention and practice. This presupposes that the time will arrive when the objective of computer based music shifts, under the endless weight of production, to the composition of music systems that explore the potential of sound from an alternate perspective.

However the process is implemented, at a fundamental level it is a scheme or logical design applied to action that re- 
sults in a desired outcome. That it can be represented in sound, an extremely temporal and visceral substance, is a very tempting and traditional objective. Yet sonic instantiation is but a fleeting indicator of the potential of any sound system. The design of the process is not illuminated by what amounts to a sonic glance.

\section{DATAESTHETICS}

Data, as it is understood in our computer-driven society, has various states and values. It is a kind of currency. An array of actions can be taken on it that alter its state and value at any given time. In a musical sense, data that is a composition process (a computer program or MIDI file) is different from data that is a musical work (audio signal) and a critical element to the difference is the value of the data as it is without additional modification or processing. The difference between a computer program and an audio sound file is obvious, but not in the same sense as a musical score is different from a performance. A process may be driven by data itself, which, in turn, may be the output of earlier processes. The resulting audio signal, which we eventually call music, may be only a snapshot of something more complex musically but that cannot be entirely or adequately expressed in a single or several musical instances. The data is, in a sense, a musical culture-a culture around which the musical signal is a kind of momentary communication channel to the outside world.

A vast amount of music today eventually gets represented in a numerical form. The ultimate objective is that this music should be heard, but every encoding is not necessarily in the final or intended form for presentation. To gain some insight into the magnitude and complex nature of "data" in general, chapter nine, "datapocalypse," of Erik Davis's text [12] is worth reading. We understand today that data is powerful, important and evanescent. Davis informs us of the historical and religious intensity that is imbued in data. It can be easily understood that to control and manipulate it is more than just a symbolic act. It represents real power. The power of musical data (the audio signal) is not always in the sound, whether completely or partially presented, but also in the ability to do something with the data.

Music, as data, as a mirror of our society, can be manipulated like all other data today, and this is a powerful creative act. It is impossible to believe that music in this coded form, in its abundance and diversity, will not become, at least in one incarnation, expressed in a universally codified form-the music of musical data. That this music will evolve from sampling (which is already extensively done) seems possible, though already passé. A more dramatic alternative would be that of a music based on strategies of processing other musical or even non-musical data or even the detritus of other music processes. This suggests that a backlash to the intense pursuit of recorded or computer-managed sound is possible not by returning to playing the acoustic guitar, but by turning the process on itself to achieve highly mutated forms or non-media-acceptable forms.

Another interesting possibility is that a contrary position be adopted. Rather than obsess about musical data, it becomes possible to ignore it or at least defer concern for it until later-lose the data. This is a challenge, as we have been well indoctrinated in the necessity of saving regularly and often. Backing up is a subconscious act, once the horror of loss is experienced. Once upon a time, music was not considered this way. It was not data to be treated in a unique or singular way on its own but performed, interpreted and presented as social information.

\section{PROJECTIONS}

One thing that has become a little clearer, and I think is a point of this essay, is that the design and construction of processes that cast sound into musical ideas is not about the reduction of music to "perceptual datum," but rather a means of thinking through music with an initial and, perhaps, deliberate absence of sound [13]. The question is, of course, why? Well, technology has made sound production too easy in some respects. But, more than that, it is the idea of approaching composition in silence. True, with technology, if we can't hear the sound, it is difficult to tell whether it is any good or not.

But that reduces "good" to a surfacelevel experience. I think that constant listening may not be the way to produce a new kind of music or a music that is perceptually different from what is produced now. The freedom of technology certainly looks like a tendency towards homogeneity these days.

Of course, composition without prelistening can be done now by going out into the wilderness and recording the environment directly, without edits, then releasing that as an untouched continuous recording experience [14]. The same thing could be fabricated as a process on the computer.

What I think is at issue here is that electronic technology has directed and indeed is probably continuing to direct the listening experience away from what has traditionally been a unique cognitive experience. It is now possible to concentrate, without distraction, on sound and let the context determine the musicality or lack thereof. It is also now possible and very common to augment sound with (or vice versa-that is, use sound to augment) other unrelated sensorial experiences (image, movement, context) that have their own narrative agendas. Recording technology introduced the practice of private listening as a solitary emotional experience. But in the past two or three decades, recordings have been used for mass emotional/sensorial experiences in the dance culture in particular, reintroducing a musical/cultural dynamic of increasing sophistication, manifest and dependent on evolving audio technology. Such a demand for music in presentation and production, coupled with the impetus that technology has had over the past 10 years at least, has begun to incline composers towards visions of "automatic" music. While this view of the "logical progression of technology" [15] has been frequently challenged, it is a cultural theme persistently espoused in much contemporary electronic music even if not actually practiced. The "Motorific" pulses, metallic beats and unnerving synthetic sounds are more often reminiscent of machines (the English duo Autechre is a good example here) than of the natural vibrations and resonances of traditional instruments. By imposing silent composition or a music of process, it seems possible to approach a future of technological composition that is not only unique but more closely aligned to a new sonic aesthetic.

\section{Acknowledgments}

I owe a debt of gratitude to Eliot Handelman and Carlos Palombini for their critical insight and comments, for which this essay is much the richer.

\section{References and Notes}

1. Leonard B. Meyer, Music, the Arts and Ideas, 2nd Ed. (Chicago, IL: Univ. of Chicago Press, 1994) p. 328.

2. Milton Babbitt, Words about Music, Stephen 
3. Joel Chadabe, Electric Sound: The Past and Promise of Electronic Music (Englewood Cliffs, NJ: PrenticeHall, 1997) p. 273

4. James Pritchett, The Music of John Cage (Cambridge, U.K.: Cambridge Univ. Press, 1994) pp. 151-152.

5. Pritchett [4] p. 151

6. Pritchett [4] p. 151.

7. Carlos Vicente de Lima Palombini, Pierre Schaeffer's Typo-Morphology of Sonic Objects, Ph.D. diss. (Durham, U.K.: University of Durham, 1993) pp. 181-182.

8. Eliot Handelman, Music as Secondary Conscious ness: An Implementation, Ph.D. diss. (Princeton, NJ: Princeton Univ. Press, 1991) p. 61 n. 11.
9. Handelman [8] p. 1.

10. Kodwo Eshun, "Circonium: Future Sound of Berlin,” The Wire, No. 169 (March 1998).

11. Donald N. Norman, The Invisible Computer (Cambridge, MA: MIT Press, 1998) p. 250.

12. Erik Davis, TechGnosis (London: Serpent's Tail, 1998) p. 253.

13. Alistair M. Riddell, Composing the Interface, Ph.D. diss. (Princeton, NJ: Princeton Univ. Press, 1993).

14. Alistair M. Riddell, "Music in the Chords of Eternity," in Contemporary Music Review 15, Part 1, pp. 29-47 (1996). Note also such CDs as Les Gilbert's Kakadu Billabong.

15. Martin Heidegger, The Question Concerning Tech- nology and Other Essays (New York: Harper Torch Books, 1977). Note Heidegger's discussion on "revealing" and "bringing forth." Here there is the implication of "progression," because the processes of "revealing" or "bringing forth" are constantly required and in demand, which has perhaps fueled a broad-spectrum notion of "progress" as an axiomatic state for all contemporary technology.

Alistair Riddell studied music and computer science at La Trobe University in Australia and holds a Ph.D. in composition from Princeton University. He is currently a freelance artist working in the area of microcontrollers and performance-sensing systems.

\section{Call for Bibliographies to be posted on Leonardo On-Line}

We are seeking bibliographies related to art, science and technology for posting on-line. Types of bibliographies might include (but are not limited to) reading lists for classes and courses that might be of interest to other educators; detailed bibliographies on a specialized topic (e.g. Art and the Internet); bibliographies of the works of single authors of interest to our readership (e.g. Rudolf Arnheim).

If you are interested in publishing a bibliography on Leonardo On-Line, contact us via E-mail: <isast@sfsu.edu> with a one- to two-paragraph description of the bibliography. This description should provide the title of the bibliography, definitions of any specific terms (e.g. "Telecommunications art"), and brief details of what the bibliography encompasses (e.g. "a listing of books, journals and theses published from 1921 to 1945 ”). It is also helpful to indicate where interested persons can find more information. We will publish this descriptive paragraph along with the bibliography if it is accepted for publication on-line; we will also publish the paragraph in the print journal Leonardo, pointing to the web site for the full bibliography.

If your bibliography is accepted for publication, we will ask you to send it along with the descriptive paragraph(s) to us on computer disk (PC or Macintosh), along with a hardcopy version for use in referring to formatting and accents.

If you have a bibliography already available on-line, send us a description and we will point to the bibliography, if appropriate. 